lapsed and became pulseless, so that it was evidently impracticable to attempt to remove the sac, which was therefore washed out, rapidly packed with several strips of iodoform gauze, and the wound partially sutured. Subcutaneous infusion of normal salt solution was then performed, and under this, and stimulants given subcutaneously and by the rectum, the patient gradually came up, and by evening had a pulse of 140. During the next few days he did well, and the course of the wound was aseptic. The pathologist's report, by Dr. F. B. Mallory, was as follows :

Microscopically the tumor is very cellular. It is largely made up of long, narrow, branching canals or spaces, lined for the most part with low, flattened cells; in places, however, the cells are taller and approach the cuboidal shape. The canals contain an almost perfectly homogeneous material. In parts of the tumor the cells are packed very closely together, so that little or no evidence of spaces can be made out, and the cells assume more or less of a spindle shape. The connective tissue stroma is slight in amount, but contains many dilated lymph spaces, which, like the canals first mentioned, contain a homogeneous material, and in places many lymphoid and plasma cells.

Diagnosis.- Lymphangiosarcoma, or perhaps better, lymphangio-endothelioma.

We had here to do with a tumor of which the rapid growth, the vascular appearance of the covering sac, the macroscopical appearance, and the microscopical examination confirmed the diagnosis of malignancy. It was also in the highest degree probable that we had not accomplished its complete removal, both because we had not excised the fibrous sac, and had perhaps left some fragments of the jelly-like contents adherent to the interior of the sac. Therefore, in view of the probability of recurrence, it was determined to try the erysipelas and prodigiosus toxins of Coley. On March 14th, the patient being in fair condition, and the wound reduced to a clean granulating sinus with slight serous discharge, 1 minim of the serum was given. It was gradually increased to 10 minims, but on April 14th, as the patient, who had developed a right femoral phlebitis, had a slight cough and pain in the chest, seemed in a critical condition, and as several small abscesses had developed at the site of the injections, it was deemed wiser to discontinue the administration of the toxins.

After this the patient made a gradual recovery, left the hospital on April 24th, and after spending the summer in western New York, returned to Boston restored to health, and resumed his employment as a salesman in one of the large department stores. He has been regularly engaged in his employment since, has gained markedly in weight and strength, and suffers only from swelling of the right leg, which is undoubtedly one of the results of the phlebitis which followed the operation. There is no evidence of the recurrence of the tumor at the present date, two years after the operation.

The case is reported on account of the rarity with which a tumor of malignant macroscopical and microscopical appearance is cured by operation, and goes to show that all tumors of malignant appearance and rapid growth are not, at least, actively malignant. It does not seem to me probable that the treatment by the mixed toxins had anything to do with the non-recurrence in this case, as, owing to its apparent bad effect upon the patient's general condition, the toxins were given only in small amounts and for a comparatively short time.

VOMITING OF PREGNANCY. - SUSPENSION OF PREGNANT UTERUS. - EXTRA-UTERINE PREGNANCY. - OPERATION FOR FIBROIDS.

REPORTED BY J. OSWALD VOGEL, M.D., From the Service of Frederick William Johnson, M.D.

Case I. Vomiting of Pregnancy.-Nellie C., twenty-one, single. Entered the medical service of the Carney Hospital, September 28, 1900, with the following history: For the past four months has had poor appetite, with occasional nausea and belching after eating. Ten days ago the patient vomited in the morning and several times during the day. There was no blood in the vomitus. The vomiting has continued ever since, coming on both during the day and during the night. Has lost about 8 pounds, although the appetite is fair. Bowels constipated. Physical examination was negative throughout. The white count was 8,200 . The urine was acid, of a specific gravity of 1,012 ; no albumin, no sugar. Since entrance the patient has vomited repeatedly, irrespective of time and feeding. No blood in vomitus; no pain ; temperature and pulse normal; no epigastric pain or tenderness; no sign in breasts of pregnancy. Rectal feeding was ordered. By mouth oxalate of serium and bismuth subnitrate, and by the rectum $1 \frac{1}{2}$ grain of cocaine were given. No effect was produced on the vomiting.

October 3d. Dr. Johnson found the uterus enlarged and somewhat to the right side of the pelvis. Cervix soft; violet color of vagina well marked. The diagnosis of probable pregnancy was made.

October 6th. In spite of every variety of treatment, the vomiting still continued, everything taken by the mouth being promptly rejected. The patient first menstruated at fifteen. Regular every four weeks until she came to this country in November, 1899, when she did not menstruate for two months. Was then regular up to June, 1900, when she did not flow for six weeks. Was unwell in July and August; but has not flowed any since August 20.

October 11 th. When etherized, a positive diagnosis of pregnancy was made. The cervix was slowly and carefully dilated to the width of an inch. Then the cervical canal was thoroughly painted with Churchill's tincture of iodine from external os to inside internal os.

October 12th. Some vomiting from the ether. On rectal feeding.

October 13th. Vomited once. Rectal feeding stopped. Semi-solid food given.

October 15th. Was put on house diet.

October 18th. No trouble in retaining all kinds 
of food. The cathartic pill taken at night caused vomiting.

October 24th. No vomiting since the 18th. and no vomiting of food since the 15th. Discharged well.

This is the second case of the persistent vomiting of pregnancy relieved by this method of treatment by Dr. Johnson.

CASE II. Suspension of pregnant uterus.Mary F., twenty-eight, married. Entered Carney Hospital November 6th, and was operated on November 8, 1900. A diagnosis of three months' pregnancy complicated with an ovarian cyst was made by her family physician, Dr. G. N. P. Mead. An incision 4 inches long was made to the right of the median line, through the right rectus muscle. The intestines were found adherent to the posterior surface of the uterus on the left. A cyst of the right ovary with twisted pedicle was found adherent to the posterior surface of the uterus and to the rectum. This cyst was the size of an orange, and was filled with dark, inky-looking material, apparently due to hemorrhage from the cyst wall. In separating the adhesions, the cyst was ruptured. The sac with right tube was removed, the tube being dissected out of the horn of the uterus, as has been Dr. Johnson's custom for years. The uterus was the size of a three months' pregnancy. The left ovary, the size of a hen's egg, was so incorporated in the cyst wall that it appeared part of it, and it took some time to decide from which side the cyst grew. The diseased portion of the left ovary was removed, leaving a rim of apparently healthy ovarian tissue, which was sewed over and over with catgut. The uterus was then suspended, after Kelly's method, with two sutures of kangaroo tendon. A quart of normal salt solution was poured into the peritoneal cavity. The abdominal incision was closed (as has been Dr. Johnson's custom for years) layer by layer, with animal ligature material, except the skin, which was united with silkworm gut. The convalescence was uneventful, and she was discharged well twenty-three days after the operation. Pregnancy has continued up to the present day with no discomfort.

This case is reported on account of the unusual procedure of suspending the uterus three months pregnant, and on account of the complication which existed with the pregnancy.

CASE III. Extra-Uterine Pregnancy. - A. N., thirty-three, married. No children. One abortion at four months, seven years ago. Menstruation began at sixteen; always regular; flows three days; uses but three napkins. No dysmenorrhea. Peritonitis lasting one month followed the abortion seven years ago. No leucorrhea. Menstruated last, November 24, 1900 ; flowed the usual amount; no pain. December 25, 1900, at 2 A. M., was suddenly taken with pain in the lower abdomen. The pain was not confined to either side, and lasted several hours. Returned in forty-eight hours very severely and lasted two hours. These pains returned, off and on, for several days, requiring the use of morphine. Was never entirely free from discom- fort in the abdomen. Was kept quiet in bed from December 27, 1900. until she was removed to the hospital January 25, 1901.

January 6th. A dark, bloody discharge began from the vagina, and continued up to the time of the operation. Often soaked through three napkins a day.

Nausea and vomiting were present during the interval from December 27 th to January 25 th.

Examined January 25, 1901. There was a dark brown, non-offensive discharge from the vagina. Uterus enlarged and in good position. Fibroid at the fundus extending toward the right. Sensitive mass, with boggy feel, behind and to the right of the uterus. Temperature normal; pulse 90 .

Operation. - Considerable tissue was curetted from inside the uterus. On opening the abdomen, the right tube was found dilated half way between its uterine and fimbriated ends to the size of a pullet's egg, and with lymph and partially organized blood around it, made a mass the size of a small orange. The tube was unruptured; the escape of blood had been from the fimbriated end. The right tube and ovary were removed, the tube being dissected out of the horn of the uterus in the usual way. The parietal peritoneum was thickened, considerably injected, and in places the intestines were adherent to it. The bladder was adherent to the intestines, the ileum and cecum adherent to each other and to the iliac fossa. The appendix was hypertrophied, covered with adhesions, and adherent to the cecum and to the gestation sac. The mesosalpinx was greatly thickened. On the left side there was old pelvic peritonitis. A fibroid the size of a goose egg was situated in the fundus. This was enucleated, and the opening in the uterus closed with catgut. The appendix was inverted into the cecum and the edges stitched over with over-andover suture of plain catgut. The uterus was suspended after Dr. Kelly's method. A pint of normal saline solution was poured into the abdominal cavity, and the abdominal wall was sewed up in four layers. The patient made an uneventful recovery.

CASE IV. Operation for fibroids. - After a long search, I can find but 2 cases similar to this one on record. One was reported by Dr. George H. Washburn, of Boston, and the other by Dr. Howard A. Kelly, of Baltimore. The unusual condition was due to the fact that the fibroids originated from the cervix and lower segment of the uterus. As they developed they grew downward into the pelvis, and as they filled this space they gradually pushed the uterus up, so that at the time of the operation in this case, it reached to the umbilicus, riding on the top of the mass, and reminding one of the howdah on the back of an elephant. In front and behind, the tubes, ovaries, round ligaments and uterus were visible and of normal size and appearance. From about the cervical junction a mass spread downward, filling the pelvis and containing forty-three separate fibroids.

E. W., thirty-three, married. Was curetted four years ago for hemorrhage. Menstruation is 
regular every four weeks; but of late there has been an increase in both the duration and the quantity of the flow. There is severe dysmenorrhea, beginning two days before the flow. The pain is in the back and lower part of the abdomen, and continues throughout the flow. Between menstrual periods there is a constant backache, with sagging and dragging down. For five years the patient has been able to feel a hard mass in the lower part of the abdomen. Has never had any children. Feet swell at times. Urine normal. On vaginal examination a round, smooth mass was found filling the pelvis. No cervix could be found. An incision was made, in the usual way, to the right of the median line through the right rectus muscle, about six inches in length. No cervix could be found, nor was it seen at any time during the operation, it having been so drawn out and incorporated into the fibroids that it was indiscernible. Extending out on either side underneath the peritoneum were what appeared to be two large masses of fibroids filling the pelvis. The mass on the left extended into the left broad ligament to the pelvic wall. The blood supply to the uterus was completely shut off, then the large mass was enucleated posteriorly. 'This mass extended beneath the uterus over to the symphysis. Then another mass on the left, extending out to the pelvic wall, was enucleated. This was of the size of a large orange, was of a very soft consistency, and was made up of a great number of small tumors. After emptying the pelvis of fibroids, a hysterectomy was done. The wound was closed, layer by layer. The patient made an uninterrupted recovery.

\section{Agroical Hrogregis.}

\section{PROGRESS IN PATHOLOGY.}

BY JAMES H. WRIGHT, M. D., BOSTON. Director of the Clinico-Pathological Laboratory, Massachu-
setts General Hospital.

MALARIA AND MOSQUITOES.

The Italian observer Grassi has recently stated that, as a result of his studies in Italy, the geographical distribution of malaria and certain species of anopheles are coincident, and that these species of mosquitoes are not found in non-malarious districts. He also states that this coincidence is probably true for other parts of the world. Celli ${ }^{1}$ has recently denied these conclusions, for he found anopheles in healthy situations in Italy which had never been malarious. More recently Nuttall, Cobbett and StrangewaysPigg ${ }^{2}$ have published an elaborate study of this question, based upon observations in England. Although malaria has apparently disappeared from England, they find three species of anopheles, not only in districts which were formerly malarious, but also in places in which malaria has never been known. The conclusion of Grassi, therefore, that anopheles are not to be found in non-malarious districts, is abundantly disproven, and it further appears that the disappearance of malaria from England is not dependent upon the absence of anopheles.

The disappearance of malaria from England is considered by them as probably due to the following causes: (1) A reduction of the number of mosquitoes consequent upon the drainage of the land; (2) the reduction of the population in infected districts as the result of emigration about the time when malaria disappeared from England; (3) the use of quinine.

The first mentioned cause seems to the authors to have been chiefly operative. They are inclined . to think that there may be another intermediary host for the malarial parasite besides man, and that this host may have become extinct in the lowlands where it is known that the fauna and flora have altered. As a further result of their observations they conclude that the numerical distribution of the anopheles is more important than their geographical distribution. The existence of anopheles in known malarious districts, they think, will explain the occasional occurrence of malaria in out-of-the-way places without making it necessary that malaria-bearing mosquitoes have been freshly imported, for under suitable conditions of temperature and the requisite number of anopheles, a malarious subject coming from other parts might well infect the local insects which in turn would spread the infection to healthy persons.

SARCOMA OF THE INTESTINE IN THE ILEOCECAL REGION.

Blaul $^{3}$ reports 2 cases of sarcoma in this situation, and summarizes 6 other cases which he has found in literature. All 8 cases were round cell sarcoma, with a relatively small amount of stroma. The tumor formations took the form of a marked thickening and infiltration of the intestinal wall, but without narrowing of the lumen of the intestine, except in 1 case. With the exception of this single case no symptoms of intestinal obstruction were observed.

The author points out that the absence of symptoms of obstruction may be of clinical significance in the differential diagnosis from carcinoma and tuberculosis. In the majority of cases the tumor ceases at the peritoneal surface, yet it may happen that this may be broken through and an enormous tumor development occur external to it. Adhesions to neighboring structures occur, but are not the rule. The production of metastases varies. In general it comes early, and may involve a great number of organs. The mesenteric and retroperitoneal lymphatic glands are usually the seat of secondary growths.

$$
\text { THE STRUCTURE OF CARCINOMa. }
$$

Peterson ${ }^{4}$ thus summarizes his study of carcinoma of the skin by reconstruction methods and serial sections.

3 Arch. Patholog. Anat. u. Physiol. u. Klin. Med. Bd. 162, H. 3. 4 Verhandl. der deutsch patholog. Gesellsch. auf der 72 Versamm lung deutsch. Naturforscher und Aerzte zu Aachen vom 16 to 22, September, 1900 . 